\title{
THEORETICAL STUDY OF POLARIZATION EFFECTS IN LATERALLY MODULATED QUANTUM WELLS
}

\author{
U. BOCKELMANN \\ Walter Schottky Institut, Technische Universität München \\ Am Coulombwall, 8046 Garching, Germany
}

\begin{abstract}
Polarization anisotropies of luminescence spectra caused by a potential modulation in the quantum well plane are discussed. Any lateral potential induces heavy hole light hole mixing. This leads to lateral polarization dependence of the interband transition rates if the confinement potential is not symmetrical with respect to rotations by 90 degrees around the quantum well growth axis. We present some numerical results for quantum wire arrays and discuss to what extent the present theory can be compared to experimental results.
\end{abstract}

PACS numbers: 78.66.-w

\section{Introduction}

The electronic and optical properties of quasi-two-dimensional semiconductor heterostructures have been treated extensively in several reviews [1-3]. Throughout the last years, quasi-one-dimensional (quantum wires) and quasi-zero-dimensional (quantum dot) structures have attracted increasing interest. A widely used technique to fabricate such structures is the lateral patterning of quantum wells. The addititonal lateral modulation can lead to dependence of the optical interband transitions versus linear light polarization which is absent in unpatterned quantum wells. There are two different causes for such lateral polarization anisotropy. First, lateral confinement induces valence band mixing, which gives rise to polarization dependence of the interband matrix element. This effect of electronic origin has been studied theoretically for quantum wires with different lateral confinement potentials [4-8] and for quantum dots [9]. The influence of a magnetic field has been also treated [10]. The other source of anisotropy is related to the spatial variation of the dielectric constant, created for instance by etching a wire pattern through a quantum well structure. This leads to polarization dependence of the local electromagnetic fields and with that to anisotropy of electromagnetic origin [11].

The present notes mainly focus on the polarization effect of electronic origin. In Sec. 2, we discuss the effect of a lateral modulation on the valence band structure 
and on the interband transition rates. We do not assume any particular shape for the lateral potential but discuss the importance of the symmetry and of the strength of the lateral confinement in general. In the third Section numerical results are presented for quantum wire arrays defined by a periodic lateral potential. In the concluding discussion we briefly consider excitonic effects, the polarization dependence of electromagnetic origin and experimental results taken from the literature.

\section{Polarization dependence of the interband matrix element}

In this section, we consider interband transitions in the dipol approximation. In this way, the perturbation of the light wave by the heterointerfaces and lateral structure is neglected. The influence of local variations of the electromagnetic fields will be discussed briefly in Sec. 4. To study interband transitions we need to know the initial and final electronic states. They are described separately by the valence band $\Gamma_{8}$ and the conduction band $\Gamma_{6}$ effective mass Hamiltonians. Excitonic effects are neglected. The conduction band states can be calculated in a single-band effective mass approximation. Here we focus on the valence band states since they are crucial for the polarization dependence of interband transitions. For a more detailed account of the theoretical framework the reader is referred to Ref. [8].

The calculation of the valence band states starts from the $\Gamma_{8}$ bulk Hamiltonian [12]

$$
H_{\Gamma_{\mathrm{s}}}=\left(\begin{array}{cccc}
H_{\mathrm{hh}} & c & b & 0 \\
c^{+} & H_{\mathrm{lh}} & 0 & -b \\
b^{+} & 0 & H_{\mathrm{lh}} & c \\
0 & -b^{+} & c^{+} & H_{\mathrm{hh}}
\end{array}\right)\left[\begin{array}{c}
3 / 2 \\
-1 / 2 \\
1 / 2 \\
-3 / 2
\end{array}\right],
$$

where

$$
\begin{aligned}
& H_{\mathrm{hh}}=-\frac{\hbar^{2}}{2 m_{0}}\left[\left(\gamma_{1}+\gamma_{2}\right)\left(k_{x}^{2}+k_{y}^{2}\right)+\left(\gamma_{1}-2 \gamma_{2}\right) k_{z}^{2}\right], \\
& H_{\mathrm{lh}}=-\frac{\hbar^{2}}{2 m_{0}}\left[\left(\gamma_{1}+\gamma_{2}\right)\left(k_{x}^{2}+k_{y}^{2}\right)+\left(\gamma_{1}+2 \gamma_{2}\right) k_{z}^{2}\right], \\
& b=\frac{\hbar^{2} \sqrt{3}}{2 m_{0}} \gamma_{3}\left(k_{x}-\mathrm{i} k_{y}\right) k_{z}, \quad c=\frac{\hbar^{2} \sqrt{3}}{2 m_{0}} \frac{\gamma_{2}+\gamma_{3}}{2}\left(k_{x}-i k_{y}\right)^{2} .
\end{aligned}
$$

Using the axial approximation for the matrix element $c_{\mathrm{n}}$ we meghect lateral anisotropy of the valence band which is weak for GaAs quantum wells gmown along the (001) crystal direction [13]. To describe lower dimensional systems we first add the confinement potential to the diagonal of $H_{\Gamma_{8}}$. We then replace the wave vector $k_{\nu}$ by the differential operator $-\mathrm{i} \partial_{\nu}$ for the directions $\nu$ withount tramslational invariance and finally symmetrize any product of non-commuting factions. The valence band wave function $\psi^{\nu}$ contains four envelope functions $\boldsymbol{f}_{\bar{j}}$ andil four atomic-like host wave functions $u_{j}$

$$
\psi^{\nu}(r)=\sum_{j=-3 / 2}^{3 / 2} f_{j}(r) u_{j}(r)
$$


The functions $u_{j}(r)$ reflect the symmetry of the $\Gamma_{8}$ band edge and are given explicitely in Ref. [8]. In general, the Hamiltonian $H_{\Gamma_{8}}$ can be diagonalized numerically only, for instance by expanding it on an appropriate set of basis functions for $f_{j}$.

Let us now consider an optical transition induced by a linearly polarized light wave. Since the periodic parts $u$ of the conduction and valence band wave functions vary rapidly over the characteristic length of variation of the envelope functions $f$, the interband transition rate is proportional to

$$
\sum_{s=-1 / 2}^{1 / 2}\left|\left\langle\psi_{s}^{c}|\varepsilon \cdot p| \psi^{\nu}\right\rangle\right|^{2}=\sum_{s=-1 / 2}^{1 / 2}\left|\sum_{j=-3 / 2}^{3 / 2}\left\langle u_{s}^{c}|\varepsilon \cdot p| u_{j}^{\nu}\right\rangle\left\langle f^{c} \mid f_{j}^{\nu}\right\rangle\right|^{2},
$$

where $s$ is the spin of the conduction band state $\psi_{s}^{c}$. The overlap integral $\left\langle f^{c} \mid f_{j}^{\nu}\right\rangle$ defines the selection rules on the electron and hole envelope functions. The dependence on the polarization vector $\varepsilon$ is determined by the atomic-like dipole matrix element. We are interested in interband transitions induced by a linearly polarized light wave propagating along the quantum well growth axis (the $z$-axis). The polarization vector lies in the $x y$-plane. The symmetry of $u^{c}$ and $u^{\nu}$ implies

$$
\begin{aligned}
& \left\langle u_{1 / 2}^{c}\left|p_{x} \cos \varphi+p_{y} \sin \varphi\right| u_{3 / 2}^{\nu}\right\rangle=\frac{m_{0} P}{\sqrt{2}} \mathrm{e}^{\mathrm{i} \varphi} \\
& \left\langle u_{-1 / 2}^{c}\left|p_{x} \cos \varphi+p_{y} \sin \varphi\right| u_{-3 / 2}^{\nu}\right\rangle=-\frac{m_{0} P}{\sqrt{2}} \mathrm{e}^{-\mathrm{i} \varphi} \\
& \left\langle u_{1 / 2}^{c}\left|p_{x} \cos \varphi+p_{y} \sin \varphi\right| u_{-1 / 2}^{\nu}\right\rangle=-\frac{m_{0} P}{\sqrt{6}} \mathrm{e}^{-\mathrm{i} \varphi} \\
& \left\langle u_{-1 / 2}^{c}\left|p_{x} \cos \varphi+p_{y} \sin \varphi\right| u_{1 / 2}^{\nu}\right\rangle=\frac{m_{0} P}{\sqrt{6}} \mathrm{e}^{\mathrm{i} \varphi} .
\end{aligned}
$$

All other matrix elements are zero. The matrix element $P$ (Kane matrix element) describes the strength of the interband transition [14]. From Eqs. (3) and 4 it follows:

$$
\begin{aligned}
& \left(m_{0} P\right)^{-2} \sum_{s=-1 / 2}^{1 / 2}\left|\left\langle\psi_{s}^{c}|\varepsilon \cdot p| \psi^{\nu}\right\rangle\right|^{2}=\frac{a_{3 / 2}^{2}+a_{-3 / 2}^{2}}{2}+\frac{a_{1 / 2}^{2}+a_{-1 / 2}^{2}}{6} \\
& -\frac{a_{3 / 2} a_{-1 / 2}}{\sqrt{3}} \cos \left(\alpha_{3 / 2}-\alpha_{-1 / 2}+2 \varphi\right)-\frac{a_{-3 / 2} a_{1 / 2}}{\sqrt{3}} \cos \left(\alpha_{1 / 2}-\alpha_{-3 / 2}+2 \varphi\right),(5)
\end{aligned}
$$

where $a_{j}$ and $\alpha_{j}$ are the absolute values and phases of the overlap between the conduction and valence band envelope functions $\left\langle f^{c} \mid f_{j}^{\nu}\right\rangle=a_{j} \mathrm{e}^{\mathrm{i} \alpha_{j}}$. From Eq. (5) it appears clearly that lateral polarization anisotropy, i.e. dependence of the interband matrix element on $\varphi$, is possible only if there is a valence band mixing. Otherwise, either $a_{3 / 2}$ and $a_{-3 / 2}$ or $a_{-1 / 2}$ and $a_{1 / 2}$ (which are the heavy and light hole components of the valence band spinor, respectively) would vanish, which would lead to a $\varphi$ independent matrix element.

In the presence of a lateral potential modulation any valence band eigenstate displays a certain amount of heavy hole light hole mixing. This is because 
the off-diagonal terms $b$ and $c$ of the Luttinger-Kohn Hamiltonian (Eq. (1)) are zero only for zero $k_{x}$ and $k_{y}$ but the lateral confinement forces the envelope function to contain Fourier components of non-zero $k_{x}$ and (or) $k_{y}$. The resulting lateral polarization anisotropy increases with the amount of valence band mixing. This is expressed in Eq. (5) by the prefactors $a_{3 / 2} a_{-1 / 2}$ and $a_{-3 / 2} a_{1 / 2}$. Large lateral anisotropy effects require strong valence band mixing, which means a lateral confinement comparable to the confinement from the underlying quantum well. For technological reasons, the lateral dimension of quantum wires and dots fabricated from quantum wells are often much bigger than the quantum well width. In such structures the lateral polarization anisotropy of electronic origin is small. Even very disordered lateral potentials can induce valence band mixing and lateral anisotropy. This has been shown explicitely for the case of quantum wells with imperfect-grid insertions by Bauer and Sakàki [15].

Equation (5) describes the polarization dependence for a given pair of initial and final states. To determine the optical transition rate the sum of all electron hole pair states, that correspond to the transition energy in question, has to be performed. For an unpatterned quantum well, e.g. Eq. (5), has to be integrated over the orientation of the $2 \mathrm{D}$ wave vector in the $x y$-plane. In the following, we show that there is no lateral anisotropy of the interband transition rate when the lateral potential is symmetrical with respect to rotations by $90^{\circ}$ around the quantum well growth axis $z$. If the lateral potential is symmetrical with respect to rotations by an angle $\beta$, the unitary matrices

$$
u(\beta)=\left(\begin{array}{cccc}
1 & 0 & 0 & 0 \\
0 & \mathrm{e}^{-\mathrm{i} 2 \beta} & 0 & 0 \\
0 & 0 & \mathrm{e}^{-\mathrm{i} \beta} & 0 \\
0 & 0 & 0 & \mathrm{e}^{-\mathrm{i} 3 \beta}
\end{array}\right), \quad u^{+}(\beta)=\left(\begin{array}{cccc}
1 & 0 & 0 & 0 \\
0 & \mathrm{e}^{\mathrm{i} 2 \beta} & 0 & 0 \\
0 & 0 & \mathrm{e}^{\mathrm{i} \beta} & 0 \\
0 & 0 & 0 & \mathrm{e}^{\mathrm{i} 3 \beta}
\end{array}\right),
$$

allow one to transform the Hamiltonian corresponding to the original reference frame (labelled 0 ) into the one rotated by $\beta$ around the $z$-axis

$$
H_{\Gamma_{\mathbf{8}}}(\beta)=u^{+}(\beta) H_{\Gamma_{\mathbf{8}}}(0) u(\beta) \text {. }
$$

The spinor of the $\Gamma_{8}$ envelope functions transforms according to.

$$
\left(\begin{array}{c}
f_{3 / 2}^{\nu} \\
f_{-1 / 2}^{\nu} \\
f_{1 / 2}^{\nu} \\
f_{-3 / 2}^{\nu}
\end{array}\right)_{\beta}=u^{+}(\beta)\left(\begin{array}{c}
f_{3 / 2}^{\nu} \\
f_{-1 / 2}^{\nu} \\
f_{1 / 2}^{\nu} \\
f_{-3 / 2}^{\nu}
\end{array}\right)_{0}
$$

Equation (8) shows that the rotation modifies the relative phases of the four valence band envelope functions. The rotation does not change the set of scalar conduction band envelope functions. From Eqs. (6) and (8) it follows that a rotation by $\beta$ shifts the argument of the cosinus terms of Eq. (5) by $2 \beta$. The lateral symmetry implies that the contributions calculated in the original and in the rotated frames have to be added to obtain the rate of the optical transition. If the lateral potential exhibits a fourfold symmetry $\left(\beta=90^{\circ}\right)$ this summation cancels the $\varphi$-dependent terms of Eq. (5). In the framework of this theory there is no lateral polarization dependence for unpatterned quantum wells and square quantum dots. On the other hand, quantum wires and elongated quantum dot structures display lateral polarization anisotropy of their interband transition rates. 


\section{Numerical results.for quantum wire arrays}

In this section we present theoretical results for quantum wire arrays as they are e.g. fabricated by a laterally patterning of quantum wells. A possible model for such structures is the harmonic potential

$$
V(x)= \pm V_{1} \cos \left(\frac{2 \pi}{L_{x}} x\right)
$$

where the lateral modulation of the conduction and valence band edges is described by an amplitude $V_{1}$ and a periodicity $L_{x}$. The negative (positive) sign corresponds to the conduction (valence) band. In this way, $V(x)$ confines the electron and hole envelope wave functions at the same lateral positions (Fig. 1). Usually the lateral

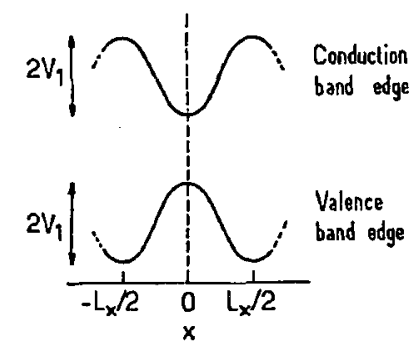

Fig. 1. Shape of the lateral model potentials.

quantization is weak compared to the one brought along by the epitaxially grown quantum well of width $L_{z}$. For structures based on symmetrical quantum wells the $1 \mathrm{D}$ valence band wave functions can e.g. be expanded in the following way:

$$
\left(\begin{array}{c}
f_{3 / 2}^{\nu} \\
f_{-1 / 2}^{\nu} \\
f_{1 / 2}^{\nu} \\
f_{-3 / 2}^{\nu}
\end{array}\right)=\left(\begin{array}{c}
\varphi_{\mathrm{hh} 1}(x) \chi_{\mathrm{hh} 1}(z) \\
\varphi_{\mathrm{lh} 1}(x) \chi_{\mathrm{lh} 1}(z) \\
0 \\
\varphi_{\mathrm{hh} 2}(x) \chi_{\mathrm{hh} 2}(z)
\end{array}\right) \mathrm{e}^{\mathrm{i} k_{y} y}
$$

The basis functions $\chi_{\mathrm{hh} 1}, \chi_{\mathrm{hh} 2}$ and $\chi_{\mathrm{lh} 1}$ are the envelope functions of the first $\mathrm{hh}$, the second $\mathrm{hh}$ and the first $\mathrm{lh}$ state of the quantum well, respectively: Another eigenstate is obtained from Eq. (10) by interchanging the first with the last and the second with the third spinor component. The resulting state is decoupled from the one of Eq. (10) since the parity operator with respect to the centre of the quantum well commutes with the IIamiltonian. At zero magnetic field, the two decoupled states have the same energy dispersion and the same interband transition rates. A magnetic field applied along the $z$-axis lifts this degeneracy.

For the harmonic lateral potentials Eq. (9), the envelope functions $\varphi(x)$ are written in the Bloch form

$$
\varphi(x)=\mathrm{e}^{\mathrm{i} k_{x} x} \sum_{K} c_{K} \mathrm{e}^{\mathrm{i} K x} .
$$

The plane-wave expansion extends over the values $K$ of the one-dimensional reciprocal lattice in the $x$-direction $\left(K=n 2 \pi / L_{x} ; n=0, \pm 1, \pm 2, \ldots\right)$. The energy 
dispersion can be folded back into the reduced Brillouin zone $0 \leq k_{x} \leq \pi / L_{x}$. The solid lines of Fig. 2 show the energies for $k_{x}=0$ and $k_{x}=\pi / L_{x}$. The dashed areas indicate the minibands of the lateral superlattice for zero in-wire momentum $k_{y}$. The widths of the forbidden energy regions created by the periodic potential
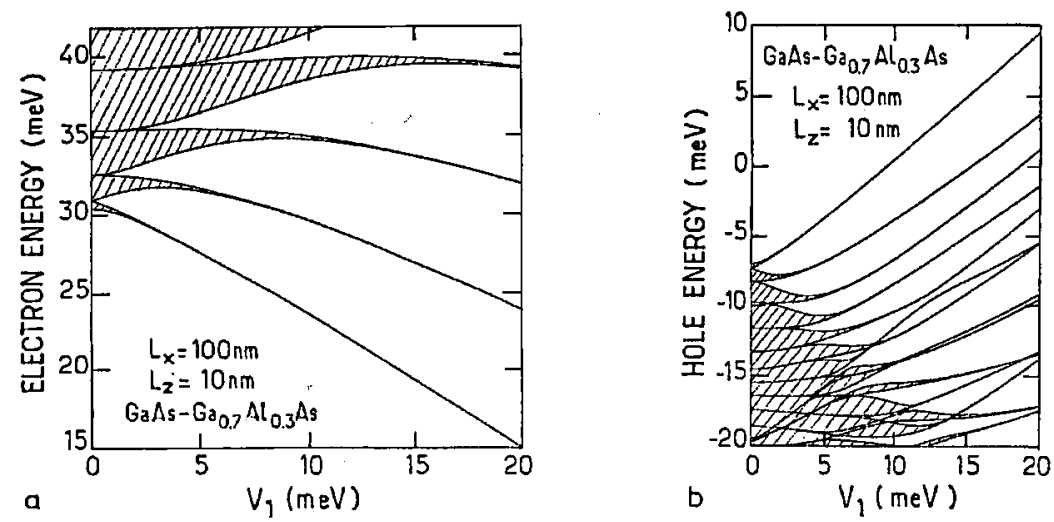

Fig. 2. Energy range covered by the miniband dispersion as a function of the amplitude of the periodic potential for (a) clectrons and (b) holes. The in-wire wave vector $k_{y}$ equals zero. Zero energy corresponds to the bottom of the bulk GaAs conduction band for (a) and to the top of the bulk GaAs valence band in (b).

increase with the amplitude $V_{1}$ and with increasing energy separation from the ground state. For large $V_{1}$, the lowest minibands become very narrow, indicating an increasingly weak tunnel coupling between adjacent potential minima. In this regime the system effectively corresponds to an array of independent quantum wires. Comparing Figs. 2a and 2b, we recognize that the hole miniband widths are smaller at a given $V_{1}$ than the electron ones. The larger hole masses lead to a stronger localization of the holes in the lateral potential. The hh-lh mixing is responsible for the nonconstant spacing of the hole levels at large $V_{1}$ and for the intricate structure deeper in the valence band.

Figure 3 shows interband absorption spectra calculated for three different amplitudes of the harmonic lateral potential. Broadening effects are introduced by replacing the energy conserving delta-function by a Gaussian function of $2 \mathrm{meV}$ width at $1 / \mathrm{e}$ maximum. The peaked structures appearing in the calculated band to band absorption arise from the quasi-one-dimensional nature of the wire eigenstates. For $V_{1}=20 \mathrm{meV}$, the peaks of the absorption spectra for lateral polarization along the wire (labelled $y$ ) and perpendicular to the wire $(x)$ correspond to the transitions h1e1 (at $7 \mathrm{meV}), \mathrm{h} 3 \mathrm{e} 1(14 \mathrm{meV}), \mathrm{h} 5 \mathrm{e} 1(18 \mathrm{meV})$ and h2e $2(20 \mathrm{meV})$. We use the notation hie $j$ to indicate a transition between the $i$-th hole and the $j$-th electron miniband. The h1e1 and $122 \mathrm{e} 2$ absorption peaks are stronger for $y$ than for $x$ polarization. The lateral anisotropy, defined as the normalized difference between the $x$ and $y$ absorption, as plotted in the upper part of the figure, is negativ. The opposite holds for the $\mathrm{h} 3 \mathrm{e} 1$ and $\mathrm{h} 5 \mathrm{e} 1$ transitions. This is because the 


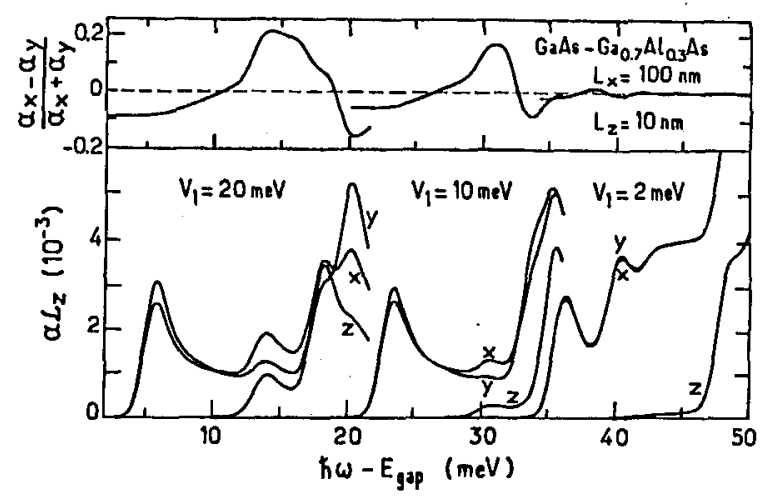

Fig. 3. Polarization-dependent absorption spectra (lower curves) and normalized lateral anisotropy (upper curves) for three different values of $V_{1}$.

first and second hole states display a dominant heavy hole character, while the third and fifth states are more light hole like (compare with Fig. 2). For quantum wire structures with electrons and holes confined in the same spatial regions a positive (negative) value of the lateral anisotropy identifies a transition involving a $1 \mathrm{D}$ valence subband of dominant th (hh) like character [8]. Figure 3 shows clearly that the lateral anisotropy increases with the amplitude $V_{1}$ of the lateral potential.

\section{Discussion}

The effect of linear light polarization on photoluminescence (PL) and photoluminescence excitation (PLE) spectra has been studied experimentally on a variety of quantum wire structures. Quantum wires fabricated by etching a lateral pattern through quantum well structures $[16,17]$ and wires directly grown on vicinal substrats $[18,19]$ or on the cleave-edge of heterostructures $[20,21]$ have been investigated this way. A quantitative comparison of the theoretical results with the experiments is difficult for a couple of reasons.

In practice the confinement potentials are barely known and the experimental spectra show important inhomogeneous broadening due to fluctuations in composition and structure size.

Excitonic effects have been neglected in the calculations. The electron-hole Coulomb interaction does not alter the spatial symmetry of the problem. Nevertheless it should influence the polarization effects of electronic origin since it modifies the electronic wave functions and energy states. Strong effects are expected especially for large quantum wires, where the exciton binding energies and the confinement energies are comparable. On the other hand, it has been shown that the anisotropy of the ground state transition barely changes due to fluctuations of the confining potential [8]. This suggests a weak exciton effect on the anisotropy of the luminescence ground state.

There is another source of anisotropy, one of electromagnetic origin. It is related to the spatial variation of the dielectric function introduced by the lateral 
pattern. A theoretical study of the light propagation through a dielectric grating shows that the local fields in the interieur of the wires depend on the polarization of the incoming light wave [11]. The magnitude of the resulting polarization effect increases with increasing modulation of the dielectric constant. Etched structures, where the different wires are separated by the ambient, display larger electromagnetic polarization effects than buried structures since the relative dielectric constant of a semiconductor is of the order of 12 while that of the ambient is 1 .

Roughly speaking, the lateral polarization dependence of large, etched (small, buried) quantum wire stuctures is mainly of electromagnetic (electronic) origin. The two causes are not decoupled in general. A full, quantitative theory of the polarization effect should include the feedback of the quantum wire on the electromagnetic wave via a frequency-dependent complex dielectric function.

In spite of the restrictions encountered above, there remains rough, qualitative agreement between experiment and theory. The experimental papers on directly grown wires [18-21] report a stronger PLE signal at the heavy hole (light hole) energy for polarization parallel (perpendicular) to the wires. For etched samples the polarization dependence is strongly affected by electromagnetic effects. Birotheau et al. normalize all spectra to their own maxima to get rid of the electromagnetic effect [17]. This way, a difference between the polarization dependence of the heavy hole and the light hole peak is observed, that increases with increasing lateral confinement again in qualitative agreement with a polarization anisotropy of electronic origin.

\section{Acknowledgments}

The presented theoretical studies have been performed at the "Laboratoire de Physique de la Matière Condensée de l'Ecole Normale Supérieure" in Paris. I wish to thank Gerald Bastard for the very fruitfull cooperation. The work has been partly supported by the "Deutsche Forschungsgemeinschaft", the "Ministère de la Recherche et de la Technologie" and the "Centre National d'Etude des Télécommunications".

\section{References}

[1] G. Bastard, J.A. Brum, R. Ferreira, in: Solid State Physics, Eds H. Ehrenreich, D. Turnbull, Vol. 44, Academic Press, New York 1991, p. 229.

[2] C. Weisbuch, B. Vinter, Quantum Semiconductor Structures. Fundamentals and Applications, Academic Press, New York 1991.

[3] Heterojunctions and Semiconductor Superlattices, Eds. G. Allan, G. Bastard, N. Boccara, M. Lannoo, M. Voos, Springer Verlag, Berlin 1986.

[4] U. Bockelmann, G. Bastard; Europhys. Lett. 15, 215 (1991).

[5] D.S. Citrin, Y.C. Chang, Phys. Rev. B 43, 11703 (1991).

[6] P.C. Sercel, K.J. Vahala, Phys. Rev. B 44, 5681 (1991).

[7] J.B. Xia, Phys. Rev. B 44, 3211 (1991).

[8] U. Bockelmann, G. Bastard, Phys. Rev. B 45, 1688 (1992). 
[9] T. Tanaka, J. Singh, Y. Arakawa, P. Bhattacharya, Appl. Phys. Lett. 62, 756 (1993).

[10] U. Bockelmann, G. Bastard, Phys. Rev. B 45, 1700 (1992).

[11] U. Bockelmann, Europhys. Lett. 16, 601 (1991).

[12] J.M. Luttinger, W. Kohn, Phys. Rev. 97, 869 (1955).

[13] M. Altarelli, in: Heterojunctions and Semiconductor Superlattices, Eds. G. Allan, G. Bastard, N. Boccara, M. Lannoo, M. Voos, Springer Verlag, Berlin 1986.

[14] E. O. Kane, J. Phys. Chem. Solids 1, 249 (1957).

[15] G.E.W. Bauer, H. Sakaki, Phys. Rev. B 44, 5562 (1991).

[16] M. Kohl, D. Heitmann, P. Grambow, K. Ploog, Phys. Rev. Lett. 63, 2124 (1989).

[17] L. Birotheau, A. Izrael, J.Y. Marzin, R. Azoulay, V. Thierry-Mieg, F.R. Ladan, Appl. Phys. Lett. 61, 3023 (1992).

[18] M. Tanaka, J. Motohisa, H. Sakaki, Surf. Sci. 228, 408 (1990).

[19] M.S. Miller, H. Wemann, C.E. Pryor, M. Krishnamurthy, P.M. Petroff, H. Kroemer, J.L. Merz, Phys. Rev. Lett. 68, 3464 (1992).

[20] D. Gershoni, J.S. Weiner, S.N.G. Chu, G.A. Baraff, J.M. Vandenberg, L.N. Pfeiffer, K. West, R.A. Logan, T. Tanbun-Ek, Phys. Rev. Lett. 65, 1631 (1990).

[21] A.R. Goni, L.N. Pfeiffer, K. West, A. Pinczuk, H.U. Baranger, H.L. Stormer, Appl. Phys. Lett. 61, 1956 (1992). 